\title{
Assessment of Effects of Amlodipine and Cilnidipine on Urea and Creatinine Levels in Hypertensive Patients-A Comparative Study
}

\author{
Sarkar S, Srivastava V* and Mohanty M \\ Department of Pharmacology, Kalinga Institute of Medical Sciences, India
}

Submission: March 23, 2018; Published: May 25, 2018

*Corresponding author: Srivastava V, MBBS, MD, Department of Pharmacology, Kalinga Institute of Medical Sciences, Bhubaneswar, Odisha, India, Email: mscutesmile.1886@gmail.com

\begin{abstract}
Background: Hypertension is one the leading cause of chronic kidney disease (CKD). The physiologic and pathologic renal changes in essential hypertension often precede changes identifiable in other organs, but whether they precede or follow the onset of the hypertension itself has not been determined. According to AHA and JNC VIII calcium channel blockers are first line drug in treatment of hypertension. The equipotent antihypertensive effect of Cilnidipine and Amlodipine in their equivalent dose has been demonstrated in number of studies.

Objectives: To compare and evaluate the effects of CCBs Amlodipine and Cilnidipine on renal parameters like urea and creatinine amongst hypertensive patients.

Methods: Total 258 patients were screened, examined and enrolled as study participants during that period. The enrolled patients were then divided as (1) Hypertensive patient ( $\mathrm{n}=159)$ )-selected patients received either Amlodipine (2.5 to $10 \mathrm{mg}$ ) or cilnidipine (5 to $20 \mathrm{mg}$ ) with or without ARB. (2) Hypertensive with controlled diabetic patients ( $\mathrm{n}=99$ )-selected patients received either Amlodipine (2.5 to $10 \mathrm{mg}$ ) or cilnidipine (5 to $20 \mathrm{mg}$ ) with or without ARB along with antidiabetic medication. Serun urea and creatinine were recorded at the baseline and periodic monitoring was done at 3,6 and 12 months.
\end{abstract}

Results: Regarding reno protective effect, the elevation of creatinine was more suppressed by Cilnidipine than Amlodipine. Both of drugs have no action on blood urea level.

Conclusion: Cilnidipine improves the creatinine levels and hence is considered to have a better reno protective profile than Amlodipine amongst hypertensive patients.

Keywords: Hypertension; Amlodipine; Cilnidipine; Urea; Creatinine

\section{Introduction}

Systemic hypertension is one of the most common non communicable diseases of mankind affecting about $20 \%$ of population globally [1]. All sections of population in India suffer from the disease, with higher prevalence in urban $(30.9 \%)$ than the rural population (21.2\%). Most of the patients with early hypertension have no symptoms but a regular monitoring of blood pressure attributes to early detection of hypertension [2]. As per 2007 AHA guidelines, Calcium channel blockers are one of the first line drugs in uncomplicated hypertension [3]. According to JNC VIII guidelines calcium channel blockers are first line of treatment in both general black or non black population (including those with diabetes).

In essential hypertension, physiologic and pathologic renal changes often precede changes identifiable in other organs, but whether they precede or follow the onset of the hypertension itself has not been determined. The earliest physiologic lesion of essential hypertension is vascular, GFR is maintained; whereas total renal blood flow is reduced (increased filtration fraction). This pattern may be explained by diffuse, predominantly efferent but also afferent, vasoconstriction of all nephrons or, alternatively, by selective afferent vasoconstriction with diversion of blood away from some nephrons to maintain near normal GFR. This renal vasoconstriction is reversible and could lead to reduced pressure and flow in the post glomerular circulation, which may predispose to increased tubule $\mathrm{Na}+$ reabsorption $[4,5]$. With this background, present study was taken up to compare the renoprotective effects of Amlodipine and Cilnidipine.

\section{Aims and Objectives}

To compare and evaluate the effects of CCBs Amlodipine and Cilnidipine on renal parameters like urea and creatinine amongst hypertensive patients. 


\section{Materials and Methods}

This is a comparative, non blinded, single centred, prospective and parallel groups, observational study was conducted in medicine OPD clinic of KIMS over a period of 24 months. The study was approved by the Institutional Ethical Committee, KIMS, BBSR. Written informed consent of all patients participating in the study was obtained. Hypertensive patients on the basis of inclusion and exclusion criteria were selected for the study.

\section{Inclusion Criteria}

A. Age: $>40 \mathrm{yrs}$ to $<60 \mathrm{yrs}$; $\mathrm{BMI}>18.5$ to $<30 \mathrm{~kg} / \mathrm{m}^{2}$ (normal and pre-obese).

B. Sex: Both sex.

C. Patients with Essential hypertension of mild to moderate cases (stage I \& stage II) according to JNC7 (those SBP $<180$ and $\mathrm{DBP}<110$ ).

D. Phase of microalbuminuria. (Spot urinary albumin creatine ratio $\mathrm{ACR}<300 \mathrm{mg} / \mathrm{gm}$ ).

E. Hypertensive patients on Amlodipine (2.5 to $10 \mathrm{mg}$ ) \& Cilnidipine (5 to $20 \mathrm{mg}$ ). Or combination with ARB (in a dose equivalent to $40 \mathrm{mg}$ of Telmisartan).

F. Controlled diabetic patient (HBA1c $\leq 7)$.

\section{Exclusion Criteria}

a. Age: $<40 \mathrm{yrs}$ to $>60 \mathrm{yrs}$; $\mathrm{BMI}<18.5$ to $>29.99 \mathrm{~kg} /$ sq. $\mathrm{mt}$

b. All cases of hypertension with $\mathrm{SBP} \geq 180$ and $\mathrm{DBP} \geq 110$.

c. Patients of secondary hypertension or taking antihypertensive medicine other than additional ACEI/ARB.

d. Uncontrolled diabetes (HBA1c $>7$ ).

e. $\quad$ Serum creatinine $\geq 1.2$

f. Patient with liver disease

g. ACR $>300 \mathrm{mg} / \mathrm{gm}$ (Spot urine)

h. Patients on Pioglitazone

i. Patients with heart failure, heart block, aortic stenosis.

j. On NSAID for long term; corticosteroid and sex steroids.

\section{Patient Recruitment}

Patients with hypertension meeting the above criteria, reporting in the department of medicine between December 14 -November 15 for their treatment were enrolled in study. Total 258 patients were screened examined and were selected as study participants during that period. The study was explained to them in local language and written informed consent was obtained. The enrolled patients were then divided as

A. Hypertensive patient $(\mathrm{n}=159)$-selected patients received either Amlodipine (2.5 to $10 \mathrm{mg}$ ) or cilnidipine (5 to $20 \mathrm{mg}$ ) with or without ARB.

B. Hypertensive with controlled diabetic patients $(n=99)$ selected patients received either Amlodipine (2.5 to $10 \mathrm{mg}$ ) or cilnidipine (5 to $20 \mathrm{mg}$ ) with or without ARB along with antidiabetic medication Serun urea and creatinine were recorded at the baseline and periodic monitoring was done at 3,6 and 12 months. Urea was measured by UV kinetic method and creatinine by Jaffe's reaction.

\section{Results}

Table $1 \& 2$ [6].

Table 1: Showing the comparative analysis of "Blood Urea" level between Amlodipine and Cilnidipine treatment on hypertensive (non diabetic and diabetic) patients.

\begin{tabular}{|c|c|c|c|c|c|c|}
\hline \multirow{2}{*}{$\begin{array}{c}\text { Data Analysed at } \\
\text { Mean } \pm \text { SD }\end{array}$} & \multicolumn{3}{|c|}{ Hypertensive Patients N=159 } & \multicolumn{3}{c|}{ Hypertensive Diabetic Patients N=99 } \\
\cline { 2 - 8 } & Amlodipine N=81 & Cilnidipine N=78 & P Value & Amlodipine N=47 & Cilnidipine N=52 & P Value \\
\hline Base Line & $25.33 \pm 4.62$ & $24.83 \pm 5.31$ & $0.5214 N S$ & $24.92 \pm 5.01$ & $24.85 \pm 5.79$ & $0.9507 N S$ \\
\hline 3 Months & $25.78 \pm 4.34$ & $24.56 \pm 5.52$ & $0.1255 N S$ & $24.98 \pm 4.8$ & $24.99 \pm 5.77$ & $0.9928 N S$ \\
\hline 6 Months & $25.62 \pm 4.57$ & $24.51 \pm 5.01$ & $0.1469 N S$ & $24.79 \pm 4.57$ & $24.8 \pm 5.5$ & $0.9919 N S$ \\
\hline 12 Months & $25.95 \pm 3.86$ & $24.72 \pm 4.89$ & $0.0839 N S$ & $25.27 \pm 4.59$ & $25.07 \pm 5.4$ & $0.8435 N S$ \\
\hline
\end{tabular}

SD: Standard Deviation; NS: Not Significant; Statics applied: Unpaired t test

Table 2: Showing comparative analysis of "Serum Creatinine" level between Amlodipine and Cilnidipine treatment on hypertensive (non diabetic and diabetic) patients.

\begin{tabular}{|c|c|c|c|c|c|c|}
\hline \multirow{2}{*}{$\begin{array}{c}\text { Data Analysed at } \\
\text { Mean } \pm \text { SD }\end{array}$} & \multicolumn{3}{|c|}{ Hypertensive Patients N=159 } & \multicolumn{3}{c|}{ Hypertensive Diabetic Patients N=99 } \\
\cline { 2 - 7 } & Amlodipine N=81 & Cilnidipine N=78 & P Value & Amlodipine N=47 & Cilnidipine N=49 & P Value \\
\hline Base Line & $0.848 \pm 0.132$ & $0.85 \pm 0.141$ & $0.9095 \mathrm{NS}$ & $0.843 \pm 0.115$ & $0.852 \pm 0.122$ & $0.7286 \mathrm{NS}$ \\
\hline 3 Months & $0.859^{*} \pm 0.133$ & $0.860^{*} \pm 0.14$ & $0.9313 \mathrm{NS}$ & $0.862^{*} \pm 0.119$ & $0.862^{*} \pm 0.127$ & $0.9812 \mathrm{NS}$ \\
\hline
\end{tabular}




\begin{tabular}{|c|c|c|c|c|c|}
\hline 6 Months & $0.867 * \pm 0.133$ & $0.869 * \pm 0.138$ & $0.9236 \mathrm{NS}$ & $0.875^{*} \pm 0.119$ & $0.873^{*} \pm 0.123$ \\
\hline 12 Months ${ }^{* *}$ & $0.89 *^{*} \pm 0.139$ & $0.853 \pm 0.138$ & $0.04 \mathrm{~S}$ & $0.902 * \pm 0.125$ & $0.851 \pm 0.125$ \\
\hline
\end{tabular}

SD: Standard Deviation; NS: Not Significant; S: significant. Statics applied: Unpaired t test and paired t test.

Predetermined clinically relevant margin is change [6] in 0.08mg/dl (i.e. 10\% variation from mean baseline value of whole study population).

*-Statistically extremely significant $(p<0.0001)$ but without clinical relevant elevation in serum creatinine is seen when compared with the base line.

**-Statistically significant $(p<0.05)$ but without clinical relevance difference in serum creatinine seen while comparing Amlodipine with Cilnidipine treatment.

\section{Discussion}

Present study shows (Table 1) no significant change in blood urea after 12 months of treatment with Amlodipine (from $25.33 \pm 4.62$ to $25.95 \pm 3.86$; p 0.0563 in DM( - ) and from $24.92 \pm 5.01$ to $25.27 \pm 4.59$; p 0.3483 in $\mathrm{DM}(+)$ patients) or Cilnidipine (from $24.83 \pm 5.31$ to $24.72 \pm 4.89$; 0.7560 in DM( $(-)$ and from $24.85 \pm 5.79$ to $25.07 \pm 5.4$; p 0.5471 in $\mathrm{DM}(+)$ patients) in both diabetic and non-diabetic patients. When comparing the effect of Amlodipine and Cilnidipine no statistically significant difference in blood urea level seen till 12 months ( $\mathrm{p}=0.0839$ in DM (-) and p 0.8435 in DM $(+)$ ) of treatment (Table 1). Kaur et al. [7] observed no change of urea level after 6 wks of treatment. Masuda et al. [8] showed that there were no significant differences between Cilnidipine treatment and Amlodipine treatment in terms of BUN and serum creatinine, when the analysis was performed on the entire population. Another animal study by Konda et al. [9] showed that, "increase in BUN, plasma creatinine level and decrease in creatinine clearence were inhibited by Cilnidipine compare to vehicle."

Present study showed that serum creatinine concentrations (Table 2) at 12 months was elevated than that of baseline, in patients who were on Amlodipine treatment (from 0.848 \pm 0.132 to $0.898 \pm 0.139 ; \mathrm{p}<0.0001,95 \% \mathrm{CI}, 0.0439<0.0509<0.0579$, in DM ( - ) and from $0.843 \pm 0.115$ to $0.902 \pm 0.125$; $<<0.0001,95 \% \mathrm{CI}$, $0.0516<0.0585<0.0655$ in DM (+)) with statistically significant but without any clinical important. On the other hand the group that were on Cilnidipine treatment (from $0.85 \pm 0.141$ to $0.853 \pm 0.138$; $\mathrm{p}=0.4296$ in $\mathrm{DM}(-)$ and from $0.851 \pm 0.122$ to $0.851 \pm 0.125$; $\mathrm{p}=0.8720$ in $\mathrm{DM}(+))$ showed no statically significance as well as clinical relevance, though there was initially rising trends was seen with Cilnidipine treatment. While comparing the effect of Amlodipine and Cilnidipine no statistically significant difference in serum creatinine level was seen till 6 months of treatment, but at the end of 12 months statistically significant difference seen ( $\mathrm{p}=0.0400,95 \% \mathrm{CI}, 0.002<0.045<0.088$ in $\mathrm{DM}(-)$ and $\mathrm{p}=0.0456$, $95 \% \mathrm{CI}, 0.00033<0.051<0.102$ in $\mathrm{DM}(+)$ patients) in both diabetic and non-diabetic patients without clinical relevance also (Table 2). Above observation can be explained by the different effect of Amlodipine and Cilnidipine on intraglomerular pressure, glomerular hyperfiltration and effect on microalbuminuria (discussed later in this section). Blood pressure was wellcontrolled in both groups as discussed earlier, so it can rule out any influence of hypertensive effects on intraglomerular pressure.
According to CAO Binquan et al. [10] Cilnidipine group showed an increase in serum creatinine levels after six months of treatment and decrease to baseline value in twelve months that collaborates with the present study. The cross over study of Hatta et al. [11] "showed serum creatinine concentrations at 12 months were elevated in the group of patients who remained on their L-CCB but not in the group that was switched to Cilnidipine" goes with present study. Streeten et al. [12] showed that a significant increase in serum $\mathrm{Cr}$ in the Cilnidipine group contradict present study, but they included patients with chronic glomerulonephritis and diabetic nephropathy. Kaur et al. [7] study concluded, no change in serum creatinine after 6 wks of treatment with Cilnidipine that does not justify present study as it is short term. Collins et al. [13] showed the serum $\mathrm{Cr}$ was slightly increased in both groups, but after 1 year of treatment, (Cilnidipine from $1.27 \pm 0.58$ to $1.37 \pm 0.72$ vs. Amlodipine from $1.29 \pm 0.60$ to $1.45 \pm 0.83 \mathrm{mg} / \mathrm{dl}$ ) corobborate present study, and it is obvious Amlodipine elevate creatinine more than Cilnidipine. Fujisawa et al. [14] shows significant elevation of creatinine level after 3 months of Cilnidipine treatment in diabetic patients, present study also shows significant elevation of creatinine level after 3 months of Cilnidipine treatment in $\mathrm{DM}(+)$ patients (from $0.852 \pm 0.122$ to $0.862 \pm 0.127 ; \mathrm{p}<0.0001$ ), this is also seen in nondiabetic patients. In the renal sub analysis of CASE-J trial, the Amlodipine-treated group manifested greater numbers of renal event (i.e., doubling of serum creatinine or end-stage renal failure); [15] and the serum $\mathrm{Cr}$ concentration increased significantly at the end of the1-year Amlodipine treatment seen on study of Kumagai et al. [16] corroborate present study. Toba et al. [17] observed that "administration of Cilnidipine to the DOCA-salt hypertensive rat reduced proteinuria, normalized the levels of creatinine, creatinine clearance and attenuated glomerulosclerosis and interstitial fibrosis, as well as the expression of collagen I/IV and TGF- $\beta$, despite the absence of any reduction in blood pressure, unlike amlodipine" also goes with present study. Biron et al. [18] showed that significant serum creatinine reduction from baseline after 2 wks of treatment with Cilnidipine contradict present study. The study of Jalal et al. [19] concluded that Amlodipine reduce creatinine after $8 \mathrm{wks}$ of treatment without statically significance contradict present study.

The possible mechanisms by which Cilnidipine acts as a renoprotective in respect to anti proteinuric action are, inhibition of glomerular hypertension and hyperfiltration by decrease in 
SNS $^{68}$ and RAAS ${ }^{55}$ activation as discussed previously (on creatinine section), and protection of podocyte by its antioxidant property. In diabetes, the main mechanisms of glomerular hyperfiltration (which may underlie the initiation and progression of DN) are by, (a) increases in the levels of hormones, such as insulin-like growth factor 1 [20], atrial natriuretic peptide [21] (b) intracellular accumulation of sorbitol and protein glycosylation [22] (c) reduced C-peptide levels and increasedcyclooxygenase-2 activity (d) exaggerated tonic influences of $\mathrm{K}+$ channels on afferent arteriolar function likely act in concert with impaired $\mathrm{Ca}_{2}+$ influx responses to changes in membrane potential promote afferent arteriolar vasodilation [23] and (e) activated tubuloglomerular feedback, which is caused by increased tubular sodium reabsorption through hyperinsulinemia and hyperglycemia. Sympathetic nerve activation is not thought to be a major mechanism of glomerular hyperfiltration in Diabetic Nephropathy [24]. According to SAKURA trial ${ }^{36}$ the sympatholytic action of Cilnidipine, although mild enough to protect the nondiabetic kidney from injury, may be too weak to counteract the glomerular hyperfiltration in the diabetic kidney caused by huge afferent arteriolar vasodilation.

Present result can be explained by another pleotrophic effect of Cilnidipine also exert its renoprotective action. Lipophilicity of Cilnidipine is greater than that of Amlodipine, which implies that Cilnidipine itself can reduce oxidative stress independently in addition to its $\mathrm{N}$-type $\mathrm{Ca}_{2}+$ channel blockade action. Excess reactive oxygen species play an essential role in the development of a variety of renal diseases such as glomerulonephritis and tubulointerstitial nephritis. Indeed, in the kidney, Cilnidipine significantly inhibited the increase in NADPH oxidase-derived superoxide production, whereas Amlodipine had no effect on the activation of NADPH oxidase in the deoxycorticosterone acetatesalt rat. Also, cilnidipine elicits podocyte protection and antiproteinuric effect in SHR/ND mcr-cp rat model of spontaneous hypertension through the reduction of renal Ang II level and a subsequent reduction in oxidative stress [25]. N-type $\mathrm{Ca}_{2}+$ channels localized in podocyte have been shown to play an important role in angiotensin II-induced superoxide production, which may partly explain the renoprotective mechanisms of Cilnidipine. Antiproteinuric effect of Cilnidipine in this present study and is in part explained by its superior antioxidant activity. Lei et al. [26] conclude that cilnidipine suppressed proteinuria and albuminuria by attenuating podocyte injury andrenal nerves have a limited contribution to the cilnidipine-induced reno-protective effects in HS-UNX-SHR. Soeki et al. [27] shows Cilnidipine probably exerts a greater renoprotective effect through its antioxidative properties.

\section{Conclusion}

From this study it can be concluded that Cilnidipine has a better renoprotective profile than Amlodipne in terms of creatinine, eGFR and UACR. Hence, in hypertensive patients with renal compromise, Cilnidipine could be the better calcium channel blocker than Amlodipine.

\section{Acknowledgement}

A. Staff members of Department of Pharmacology

B. Staff members of Department of Medicine.

\section{References}

1. Krishnadas KV (2004) Text book of medicine. Jaypee Brothers, $\left(4^{\text {th }}\right.$ edn), India, 2: 651.

2. Marschall SR, Andrew MG (2003) Netter's internal medicine. Icon learning systems LLC, USA, pp. 127-128.

3. Joseph JS (2009) Hypertension, applied therapeutics, the clinical use of drugs. Mary Anne KK, Lloyd YY, Brian KA (Eds.), ( $9^{\text {th }}$ edn), Lippincott Williams \& Wilkins, USA, pp. 13-18.

4. De Leeuw PW, Kho TL, Falke HE, Birkenhäger WH, Wester A (1978) Hemodynamic and endocrinological profile of essential hypertension. Acta Med Scand Suppl 622: 5-86.

5. Goldring W, Chasis H, Ranges HA, Smith HW (1941) Effective renal blood flow in subjects with essential hypertension. J Clin Invest 20(6): 637.

6. Wannamethee SG, Shaper AG, Perry IJ (1997) Serum creatinine concentration and risk of cardiovascular disease: a possible marker for increased risk of stroke. Stroke 28(3): 557-563.

7. Kaur M, Sharma AK, Mahajan DS, Takia T, Goel D (2012) A comparative study of therapeutic effects and tolerability profile of cilnidipine versus amlodipine in mild to moderate essential hypertension. IJPSR 3(12): 5044-5055.

8. Masuda T, Ogura MN, Moriya T, Takahira N, Matsumoto T, et al. (2011) Beneficial effects of $\mathrm{L}$ - and $\mathrm{N}$-type calcium channel blocker on glucose and lipid metabolism and renal function in patients with hypertension and type II diabetes mellitus. Cardiovasc Ther 29(1): 46-53.

9. Konda T, Enomoto A, Takahara A, Yamamoto H (2006) Effects of L/Ntype calcium channel antagonist, cilnidipine on progressive renal injuries in Dahl salt-sensitive rats. Biol Pharm Bull 29(5): 933-937.

10. Binquan C, Dongyue L, Shangzhong W, Yinggui F (2010) Cilnidipine for treatment of essential hypertension accompanied with type II diabetes mellitus incorporated with kidney disfunction. China Medicine 5(6): 1673-4777.

11. Hatta T, Takeda K, Shiotsu Y, Sugishita C, Adachi T, et al. (2012) Switching to an $\mathrm{L} / \mathrm{N}$-type calcium channel blocker shows renoprotective effects in patients with chronic kidney disease: the kyoto cilnidipine study. J Int Med Res 40(4): 1417-1428.

12. Streeten DH, Anderson GH, Wagner S (1990) Effect of age on response of secondary hypertension to specific treatment. Am J Hypertens 3(5.1): 360-365.

13. Collins R, Peto R, MacMohan S, Hebert P, Fiebach NH, et al. (1990) Blood pressure, stroke, and coronary heart disease. Part 2, Short-term reductions in blood pressure: overview of randomised drug trials in their epidemiological context. Lancet 335(8693): 827-839.

14. Fujisawa T, Ikegami H, Noso S, Hiromine Y, Kawabata Y, et al. (2007) Renoprotective effect of $\mathrm{N}$-type $\mathrm{Ca}$ channel blockade in diabetic nephropathy. J Diabetes Complications 21(4): 252-257.

15. Saruta T, Hayashi K, Ogihara T, Nakao K, Fukui T, et al. (2009) Effects of candesartan and amlodipine on cardiovascular events in hypertensive patients with chronic kidney disease: subanalysis of the CASE-J study. Hypertens Res 32(6): 505-512.

16. Kumagai H, Hayashi K, Kumamaru H, Saruta T (2000) Amlodipine is comparable to angiotensin-converting enzyme inhibitor for long-term renoprotection in hypertensive patients with renal dysfunction: A oneyear, prospective, randomized study. Am J Hypertens 13(9): 980-985. 
17. Toba H, Yoshida M, Tojo C, Nakano A, Oshima Y, et al. (2011) L/Ntype calcium channel blocker cilnidipine ameliorates proteinuria and inhibits the renal rennin-angiotensin-aldosterone system in deoxycorticosterone acetate-salt hypertensive rats. Hypertens Res 34(4): 521-529.

18. Biron P, Mongeau JG, Bertrand D (1976) Familial aggregation of blood pressure in 558 adopted children. Can Med Assoc J 115(8): 773-774.

19. Jalal S, Sofi FA, Abass SM, Alai MS, Bhat MA, et al. (2010) Effect of amlodipine and lisinopril on microalbuminuria in patients with essential hypertension: A prospective study. Indian J Nephrol 20(1) 15-20.

20. Hirschberg R, Kopple JD (1992) The growth hormone-insulin-like growth factor I axis and renal glomerular function. J Am Soc Nephrol 2(9): 1417-1422.

21. Anderson S, Vora JP (1995) Current concepts of renal hemodynamics in diabetes. J Diab Complications 9(4): 304-307.

22. Sabbatini M, Sansone G, Uccello F, Antonella G, Giuseppe C, et al. (1992) Early glycosylation products induce glomerular hyperfiltration in normal rats. Kidney Int 42(4): 875-881.
23. Pamela KC (2010) The renal vascular response to diabetes. Curr Opin Nephrol Hypertens 19(1): 85-90.

24. Vallon V, Blantz RC, Thomson S (2003) Glomerular hyperfiltration and the salt paradox in early type 1 diabetes mellitus: a tubulo-centric view. J Am Soc Nephrol 14(2): 530-537.

25. Fan YY, Kohno M, Nakano D, Ohsaki H, Kobori H, et al. (2010) Cilnidipine suppresses podocyte injury and proteinuria in metabolic syndrome rats: possible involvement of $\mathrm{N}$-type calcium channel in podocyte. J Hypertens 28(5): 1034-1043.

26. Bai L, Daisuke N, Yoshihide F, Ya L, Hirofumi H, et al. (2012) N-type calcium channel inhibition with cilnidipine elicits glomerular podocyte protection independent of sympathetic nerve inhibition. J Pharmacol Sci 119(4): 359-367.

27. Soeki T, Kitani M, Kusunose K, Yagi S, Taketani Y, et al. (2012) Renoprotective and antioxidant effects of cilnidipine in hypertensive patients. Hypertens Res 35(11): 1058-1062.

\section{Your next submission with Juniper Publishers} will reach you the below assets

- Quality Editorial service

- Swift Peer Review

- Reprints availability

- E-prints Service

- Manuscript Podcast for convenient understanding

- Global attainment for your research

- Manuscript accessibility in different formats

( Pdf, E-pub, Full Text, Audio)

- Unceasing customer service

Track the below URL for one-step submission https://juniperpublishers.com/online-submission.php 